\title{
EDITORIAL
}

\section{The quality of life construct in psychiatry-the state of the art}

\author{
STEFAN PRIEBE
}

Unit for Social E Community Psychiatry, Barts and the London School of Medicine, Queen Mary, University of London, UK

This issue is on the construct of quality of life in psychiatry and the use of quality of life indicators in different fields of mental healthcare. It is not the first specially edited journal issue or book on the topic, and it will not be last one. Quality of life has become an extremely popular construct in mental healthcare and is of major interest to researchers and clinicians alike. Some of the authors of the articles in this issue have been members of the 'International Quality of Life in Mental Healthcare Research Group' that in 1999 published a book Quality of life in mental health care (Priebe et al., 1999) and contributed to a special issue of the International Fournal of Social Psychiatry on quality of life in the same year (Holloway \& Oliver, 1999). The book dealt with quality of life issues in a systematic way, beginning with chapters on the construct of quality of life and methods for assessment and ending with options for further research. The issue of the International foumal of Social Psychiatry contained a collection of papers of specific interest. This issue takes a different approach. In a somewhat systematic but not comprehensive fashion, it covers different conceptual and practical aspects of quality of life research - such as its use in non-Western cultures and individual treatment processes, its association with other related constructs - and the application of the construct in important patient groups in mental healthcare-i.e. mentally ill offenders and patients with anxiety and depressive disorders, severe mental illness and substance abuse - as well an article on quality of life in the general population.

What does this issue as a whole-that is beyond the statements of single papers - tell us about the state of the art of quality of life research in psychiatry? Approximately 20 years after the beginning of quality of life research in psychiatry, there obviously is a vast amount of literature on the subject. The number of scales that have been used to assess quality of life in psychiatric patients is enormous and increases every year. Various research groups have accumulated large sets of quality of life data. Many of the more recent studies have been longitudinal and well designed. For example, the influence of factors such as psychopathology on quality of life ratings has often been properly assessed and controlled for. These studies have provided a substantial body of knowledge on quality of life parameters particularly in patients with severe and chronic conditions. This knowledge is summarized in the articles of this issue and the reader will certainly get an idea of the progress that has been made in the field. One might argue that quality of life research in psychiatry has grown out of its infancy. Yet, at the same time, it is far from being complete. Surprisingly many of the articles identify a lack of methodologically sound and rigorous research in the area they look at and end with a conclusion that more research is needed. There are-at least-three major challenges to future research: One is conceptual. More theoretical and empirical work is needed to further develop the construct of quality of life and distinguish it from other concepts. Health-related and generic constructs need to be specified, be based on empirical data and be revised if and as necessary. Mere claims of authors as to what constitutes quality of life are to be replaced by constructs that are supported by specific theory and consistent with empirical results. This probably requires input from basic sciences such as psychology, sociology and anthropology. The second challenge is to conduct quality of life research in patient groups other than those with non-psychotic disorders of working age, being cared for in community mental health teams. The articles of Hansson, Rudolf \& Watts, and van Nieuwenhuizen et al. provide some hints on how to do this. The third major challenge is to find ways to use quality of life indicators for policy decisions and planning 
purposes, as well as in service development and individual treatment. So far, various forms of quality of life indicators have been measured in order to evaluate services and treatment methods. This, however, can only be the first step. In a next step researchers have to be concerned about what to do with the results. It should be explored whether and, if so, in what way the quality of life construct opens up the way to new interventions on an individual and service level. More knowledge on how the quality of life of psychiatric patients can be improved may or may not lead to interventions that are qualitatively different from current practice.

Progress in quality of life research - as in any other field of science-is likely to be marked by controversy, debate and struggle for consensus. All of the reviews in this issue have been written by experts. Nevertheless they take different angles and use different terminology. In the introductions they describe the origins of the quality of life construct in different ways and provide different explanations for why the construct has become popular and important. Some of the assumptions and conclusions are inconsistent if not contradictory. This may be a sign of semantic and conceptual confusion or of a productive phase of debate or both. In any case, the articles represent the state of the art in the year 2002 . Let us hope that they will be outdated soon as a result of significant further progress.

\section{References}

Holı.oway, F. \& OlIVER, J.P.J. (Eds) (1999) Quality of life and mental health services. International foumal of Social Psychiatry, 45 (Special issue), 235-320.

PrIebe, S., Olwner, J.P.J. \& KaISER, W. (Eds) (1999). Quality of life and mental health care. Petersfield: Wrightson Biomedical. 